\title{
Influence of volatiles oxidation processes on kinetics of sawdust combustion
}

\author{
Igor Donskoy ${ }^{1, *}$, Aleksandr Kozlov ${ }^{1}$, and Vitaliy Shamanskiy ${ }^{1}$ \\ ${ }^{1}$ Melentiev Energy Systems Institute, 130 Lermonova Str., Irkutsk, 664033, Russia
}

\begin{abstract}
The paper considers influence of the devolatilization on the combustion of a small woody particle at TGA conditions. Differences between woody char and raw sawdust combustion kinetics are evaluated. Gas formation kinetic parameters are estimated.
\end{abstract}

\section{Introduction}

Most of the studies devoted to the modeling of wood particle combustion assume simplifications related to the impact of kinetics of gas-phase reactions on heterogeneous reactions of fuel. For example, it is often assumed that kinetics of gas-phase reactions can be defined by a small number of kinetic equations for overall reactions that involve model substances (methane or a conventional surrogate component [1]). The reaction capability of a wood char in this case normally does not depend on the history of its formation. However, owing to the high rate of chain reaction spread in the gas medium space, the flame front of volatiles combustion can influence kinetics of heterogeneous oxidation. Active particles formed in the flame penetrate into the surface layer where they can both activate part of solid phase of already pyrolized fuel, and accelerate pyrolytic destruction of still remaining macromolecules and their fragments $[2,3]$. At the same time thermal destruction of wood macromolecules occurs primarily according to a radical mechanism, and transport of radicals from internal regions to the particle surface may be of a relay-race nature, thus forming their diffusion flow. This process in turn triggers chain mechanisms in the nearsurface flame and increases the rates of gas-phase reactions.

\section{Experimental setup and results analysis}

To study the phenomena that occur at thermochemical conversion of wood particles we used a synchronous thermal analyzer manufactured by the firm NETZSCH-Geratebau. It consists of thermal analysis unit STA 449 F1, quadruple mass spectrometer QMS 403 AEOLOS and a pulse thermal analysis unit Pulse TA. The obtained products of solid fuel pyrolysis and combustion were recorded in a range of mass numbers from 1 to 200, with 70 $\mathrm{eV}$ electron impact ionization. The mass-spectrometer signal was calibrated by pulse supply of respective calibration gases.

* Corresponding author: donskoy.chem@,mail.ru 
The initial wood composition was determined by thermal analysis: moisture content $3.47 \%$, ash content $-1.40 \%$, carbon content $-54.28 \%$, hydrogen content $-5.20 \%$, oxygen content $-40.12 \%$, nitrogen content $-0.40 \%$, and the lowest calorific value $-20.52 \mathrm{MJ} / \mathrm{kg}$. Lignin content in the organic matter makes up $34.0 \%$, tar $-5.3 \%$. The detailed technique is presented in [4].

The research involved $11-12 \mathrm{mg}$ of sawdust, about $0.2 \mathrm{~mm}$ in size. A representative sample of sawdust (pine) prepared by a standard quartering method was placed in the alumina crucible with low walls. Such a form of the crucible facilitates a uniform access of oxidizer to fuel particles. The crucible was placed in the furnace of thermal analyzer and heated from a room temperature up to $900^{\circ} \mathrm{C}$ at a rate of $10^{\circ} \mathrm{C} / \mathrm{min}$ in the argon flow with a flow rate of $80 \mathrm{ml} / \mathrm{min}$. The sawdust pyrolysis was accompanied by removal of volatiles and formation of wood char particles. Then, the sample was cooled to $100^{\circ} \mathrm{C}$, without taking it from the furnace, and further the obtained char was completely burnt in the air flow at the same heating rate. The air flow rate was $70 \mathrm{ml} / \mathrm{min}$. The other sample was heated in the air flow until complete combustion under the indicated parameters. The obtained thermogravimetric curves are presented in Figure 1. The results were calculated on a dry and ash-free organic wood matter basis.

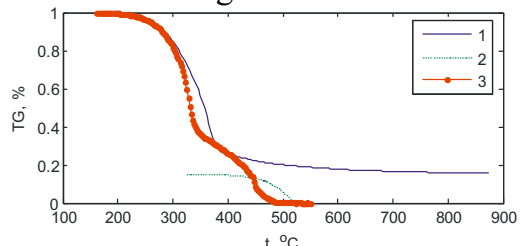

a

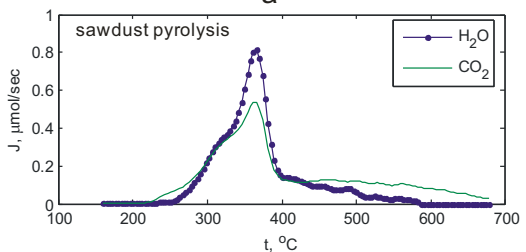

C

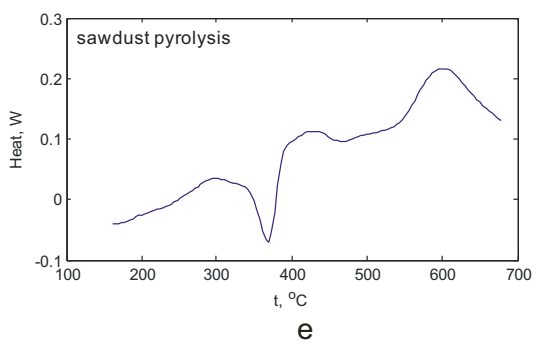

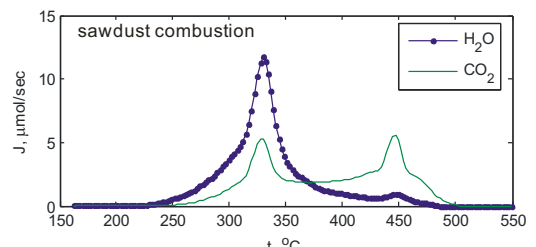

b

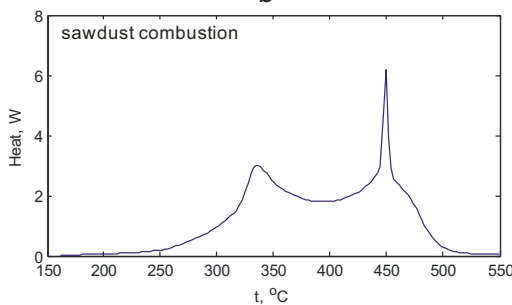

d

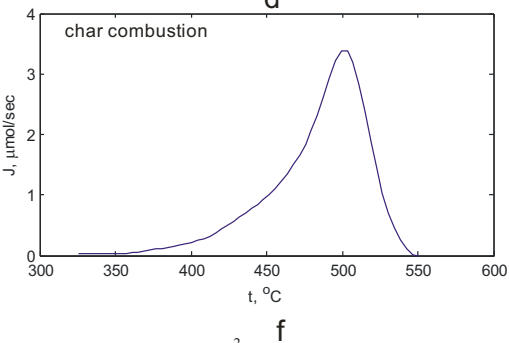

Fig. 1. Experimental curves for sawdust and char combustion.

Figure $1 a$ shows that the oxidative pyrolysis and further combustion of wood char without clearly identified pyrolysis stage occur more intensively even at lower temperatures than on the curves with preliminary pyrolysis in an inert medium. The first bend on thermogram 3 apparently corresponds to a transition from combustion of mainly carbohydrate components to combustion of lignin, and the second one corresponds to complete combustion of wood char. This is confirmed by the dynamics of $\mathrm{H}_{2} \mathrm{O}$ and $\mathrm{CO}_{2}$ formation in sawdust combustion, which is shown in Figure $1 b$. Figure $1 b$ illustrates that the maximum intensity of water formation is observed in a temperature interval of 320 - 
$350^{\circ} \mathrm{C}$, whereas the intensity of $\mathrm{CO}_{2}$ production has two peaks which can be referred to the destruction of aliphatic and aromatic structures: $320-350^{\circ} \mathrm{C}$ and $430-560^{\circ} \mathrm{C}$, respectively. Similar phenomena were observed in [5-8].

In order to assess the potential impact of organic wood matter pyrolysis on the intensity of gas-phase reactions we will consider temperature dependence of the $\mathrm{CO}$ and $\mathrm{CO}_{2}$ formation intensity in the course of pyrolysis in an inert medium (Fig. 1c). In this case the peaks of the $\mathrm{CO}$ and $\mathrm{CO}_{2}$ formation intensity occur in an interval of $350-400^{\circ} \mathrm{C}$, whereas in the oxidative pyrolysis as soon as this temperature is reached the burnout of cellulose components of the organic wood matter finishes. An analysis of the total thermal effect at oxidation of volatile components (Fig. 1d) shows that after $250^{\circ} \mathrm{C}$ their oxidation is accompanied by the formation of $\mathrm{CO}_{2}$. Pyrolysis in an inert medium (Fig. 1e) in the region of volatile yield peak values is a low-energy process. It is however worth noting that in this case we record a total effect of exothermic intramolecular oxidation with molecule restructuring and formation of volatile components, and energy consumed for the related disruption of chemical and intermolecular bonds. We can assume that the development of oxidative processes in gas phase (most probably in the near-surface layer of a fuel particle) under such a relatively low temperature is possible only in the case where generation of active particles (radicals) occurs at the destruction of macromolecules, i.e. inside the particle, where this energy consumption is compensated for by the heat of internal oxidation. Figure $1 e$ shows that it is precisely in the area near $250^{\circ} \mathrm{C}$ the total thermal effect transitions to the positive value region. The experiment that proves the emergence of radicals in gas phase at biomass pyrolysis is described in [2].

An analysis of wood char combustion after pyrolysis is made on the basis of temperature dependence of carbon dioxide formation in this process (Fig. 1f). The elementary kinetic equation that describes this process can be written as follows:

$$
J=\frac{d n_{\mathrm{CO}_{2}}}{d \tau}=k \cdot \exp \left(-\frac{E_{a}}{R T}\right) \cdot S \cdot\left(n^{0}-n\right) \cdot C_{\mathrm{O}_{2}} .
$$

Here: $E_{a}$ is activation energy, $\mathrm{J} / \mathrm{mole} ; S$ is specific area of a sample, $\mathrm{m}^{2} / \mathrm{mole} ; n$ is wood char quantity, mole; $C$ is oxygen concentration in gas phase, mole $/ \mathrm{m}^{3}$; and $k$ is the matching factor. Assuming that at the beginning of the process the area of a sample reaction surface changes little, since the total decrease in the geometrical surface can be accompanied by an increase in the surface concentration of active centers, and taking into account that oxygen concentration in the oncoming reaction flow does not change, (1) can be transformed to the form: $\ln J=a-E_{a} / R T$. Figure 7 presents a diagram of relationships between $\ln J$ and $1 / T$. Linear part belongs to an ascending branch of curve in Fig. 8. This is a range of constant activity of wood char surface $\left(\mathrm{T} \sim 360 \div 500^{\circ} \mathrm{C}\right)$. The activation energy determined from the diagram equals $119 \mathrm{~kJ} / \mathrm{mole}$.

There is a similar section in the interval of temperatures $\mathrm{T} \sim 270 \div 320^{\circ} \mathrm{C}$ on the curve constructed for oxidative pyrolysis (Fig. 8), which also corresponds to an ascending branch of a carbon dioxide formation curve for the first peak in Fig. $1 b$. In this case the process rate rises approximately 250 times (while in reality even more, since the combustion temperature in the case of oxidative pyrolysis is lower). Since the parameters of external gas phase (i.e. beyond the diffusion surface layer) in both cases differ only in temperature, such an increase in the rate can be explained by a higher energy of carbon atoms in the initial wood. However, the actual equality between the activation energies of $\mathrm{CO}_{2}$ formation at wood char combustion and at the beginning of the oxidative pyrolysis needs to be explained. We can suppose, in particular, that the internal oxidation, i.e. oxidation involving atoms of oxygen in the wood, which undoubtedly occurs at pyrolysis, hardly takes place for carbon in this case. Most likely, oxygen of carbohydrate and related 
molecules is consumed for water formation. Intensive and synchronous with $\mathrm{CO}_{2}$ release of water in this range of temperatures is seen in Figure $1 b$. After removal of water molecules from macromolecules, for example, cellulose molecules, the areas of carbon matrix that differ from steady allotropic structures of free carbon, i.e. have a higher Gibbs energy, are formed in the wood. It is precisely these areas that are attacked by the molecules of oxygen or oxygen containing radicals from a surface layer. Certainly such a mechanism does not refer, for example, to the carboxyl carbon or carbon of extractive components (tar). However, in percentage terms the concentration of such carbon is low: no more than several per cent of the total amount. In the case of pyrolysis in an inert medium (Fig. 1c), it is seen that carbon dioxide yield is almost 10 times less than in an oxidative medium. Note that nearly $65 \%$ of organic wood matter (Fig. 1a), i.e. its carbohydrate constituent, burns out before the temperature reaches $\sim 330^{\circ} \mathrm{C}$.
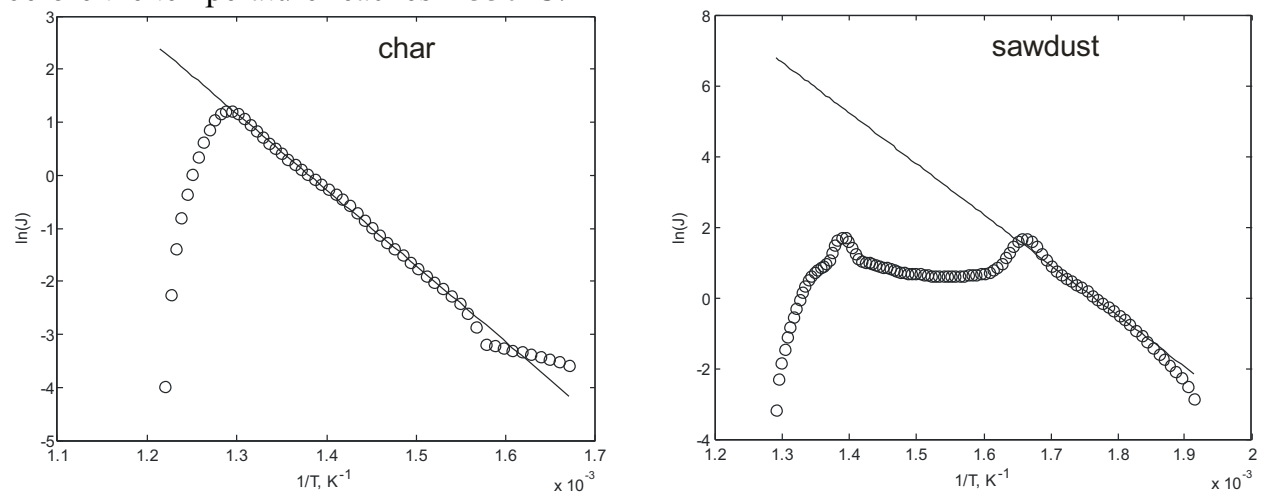

Fig. 2. Arrhenius plot for $\mathrm{CO}_{2}$ in char and sawdust combustion

An in-depth consideration of the above qualitative suppositions on the wood pyrolysis and combustion mechanisms is possible involving the tools of detailed chemical kinetics [9]. The study on the sawdust pyrolysis will further the detailed study of chemical kinetics, which will create preconditions for research into large particles where thermal inhomogeneity leads to simultaneous processes of oxidative (at the surface) and inert (intraporous, protected by Stefan flows) pyrolysis.

The work is supported by Russian Foundation for Basic Research (project No. 16-08-00694).

\section{References}

1. J. Yu, M. Zhang, J. Zhang, Proc. Comb. Inst. 32, 2037 (2009).

2. A. Kozlov, D. Svishchev, I. Donskoy, V. Shamansky, J. Therm. Anal. Calorim. 122, 1098 (2015).

3. B. Goshayeshi, J.C. Sutherland, Comb. Flame. 141, 1900 (2014).

4. A. Kozlov, D. Svishchev, I. Donskoy et al., J. Therm. Anal. Calorim. 122, 1213 (2015).

5. S. Munir, S.S. Daood, W. Nimmo et al., Bioresource Tech. 100, 1413 (2009).

6. K. Cheng, W.T. Winter, A.J. Stipanovic, Polymer Degradation Stability 97, 1606 (2012).

7. W. Wu, Y. Mei, L. Zhang, R. Liu, J. Cai, Fuel 156, 71 (2015).

8. N. Aniza, S. Hassan, M. Int. J. Mech. Eng. Sci. 10, 1943 (2016).

9. E. Ranzi, T. Faravelli, F. Manenti, Adv. Chem. Eng. 49, 1 (2016). 